\title{
BREAKING BRICKZ A 2D ANDROID GAME
}

\author{
Dhawal Gowda ${ }^{1}$ \\ ${ }^{1}$ BE Student, Department of Information and Technology, Alamuri Ratnamala Institute of Engineering and \\ Technology, Shahpur, Maharastra, India
}

\begin{abstract}
In this paper we are developing a 2-D android game which has more than 30 levels. It is a puzzle game it can be also called as a memory game. It can be played on any android device irrespective of the screen size. This game is a memory game which can improve the ability to focus of the user and help in building his concentration. This game is developed in c-sharp and using a game engine called unity.
\end{abstract}

Keywords: Android, Game, Puzzle Game

\section{INTRODUCTION}

Breaking brickz is a 2-D android game which runs on any android device of any screen size. It helps in improving the concentration power of the user, the game has 30 different levels with different puzzles each having its own difficulty this game delineated as a memory game. This game is developed in c\# (c sharp) and the game engine used is called as unity.

Gameplay: There is a box in the gameplay and the main objective of the player is to make the box reach its destination within the time allotted, there are bricks that is called as the direction bricks which contains different directions, the player has to break this bricks by touching them on the screen each brick has a particular direction necessary to move the box, some directions disappear after some time so user has to remember the directions and move the box accordingly there is also some obstacles which will hinder the path of the box the user has to avoid these obstacles.

\section{LITEREATURE REVIEW}

Gaming industry is a rapidly growing market and will on growing in the near future, with the advent of android system a new market has immerged and has open the paths to many mobile game developers .There are many games available on the google play store each having its own genre like puzzle, racing, adventure, sides scroller, action, casual etc. People play mobile game while travelling and also in their free time, people of all age group loves to play game and mobile games are daunting in this area. Every game has its life, it means that people play a particular game for some period of time and switch to next game hence every game developer gets a chance to showcase their talent and be successful. Games can be played with two screen orientation that is landscape and portrait, landscape games such as Into the dead, Clash Of Clans, Jet Pack Joyride are very famous and portrait games such as Candy Crush Saga, Bubble Witch Saga, Temple Run, Subway Surfers are very famous. Some games provide with both the screen orientation options. There are also motion games which can be controlled via motion of the mobile this is because the smart phones contains gyroscope. Gyroscope is the sensor which identifies when you tilt the screen and provides that information to the game and it works in that direction accordingly, developers access this gyroscope via programming to make the necessary changes. Racing games mostly contains motions control such as games like Asphalt and NFS they use the gyroscope to control the position of the car.

\section{OBJECTIVES}

The main objective of this project is to create a game that the user will play for years and will not lose interest in the game. There are many games on the play store each having their own genre but more than half of the games are not recognized and have a short life span of one month some games have a life span of a week and some of days, due to the introduction of android a lot of games have been created but more than half of them fail so our main objective is to create a interesting game. We will keep on adding different levels to the game so that the user will keep on indulging into the game.

\section{PROPOSED SYSTEM}

System architecture is a conceptual model that defines the structure, behaviour, and more views of a system. An architecture description is a formal description and representation of a system, organized in a way that supports reasoning about the structures and behaviours of the system. A system architecture can comprise system components that will work together to implement the overall system 


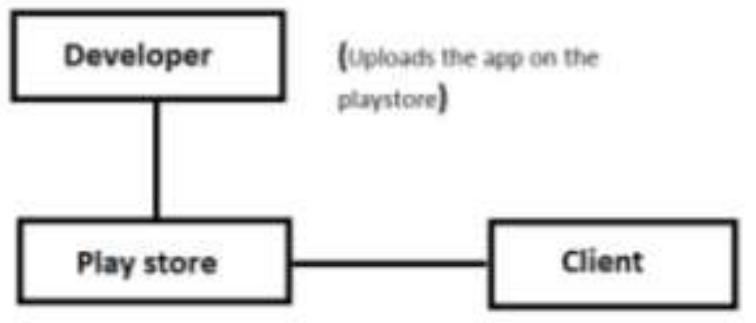

(App available gloabally)

(User downiloads the app)

Fig 1: Proposed System Architecture

There are three main components of the system Developer, Play store and Client the application's life depends on these three components

\subsection{Developer}

Developer is the person who is the creator of the application. He implements his ideas into the game and can use different references and software for this purpose. The developer can be an individual or an organization. The developer after successfully creating the software interacts with the play store to upload the game, he needs to pay a one-time fees for buying the developer account at google play store and after that he can upload numerous games. He can also make updates of the same application depending on the scenario.
He has the authority to publish or unpublish a game whenever he wants.

\subsection{Play Store}

Play store acts as the middle man between the developer and the client. Developer uploads the game on play store and client downloads the game via play store. There are numerous application on the play store hence application often gets lost or unrecognized for this play store offers advertising, developer needs to play some amount to increase the download of their application. Play store also provides the tag of top developer to the developers creating mesmerizing games and also the Editor's Choice to some extra ordinary games. Play store has the authority to unpublish the application who violate the terms and condition.

\subsection{Client}

Client is the person who download the game form the play store. Client can also rate the games which he likes and also comment on it. Client can report inappropriate games and the play store will take strict action regarding this.

\section{SYSTEM DESIGNS}

Here we will show you the application interface that have been designed, and named by its function in the system.

\subsection{Loading Screen}

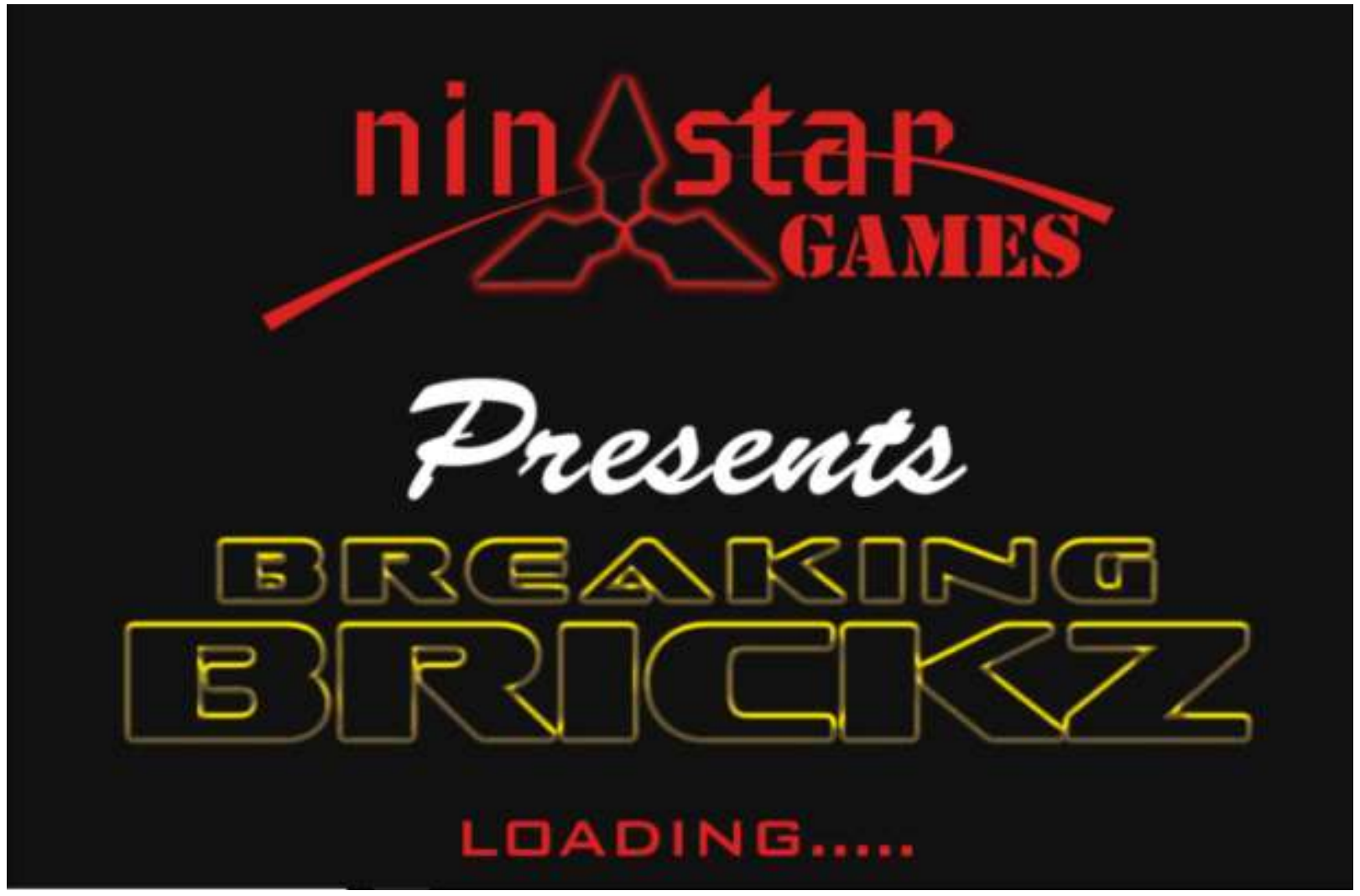

This image will be displayed while the screen is loading 


\subsection{Home Screen}

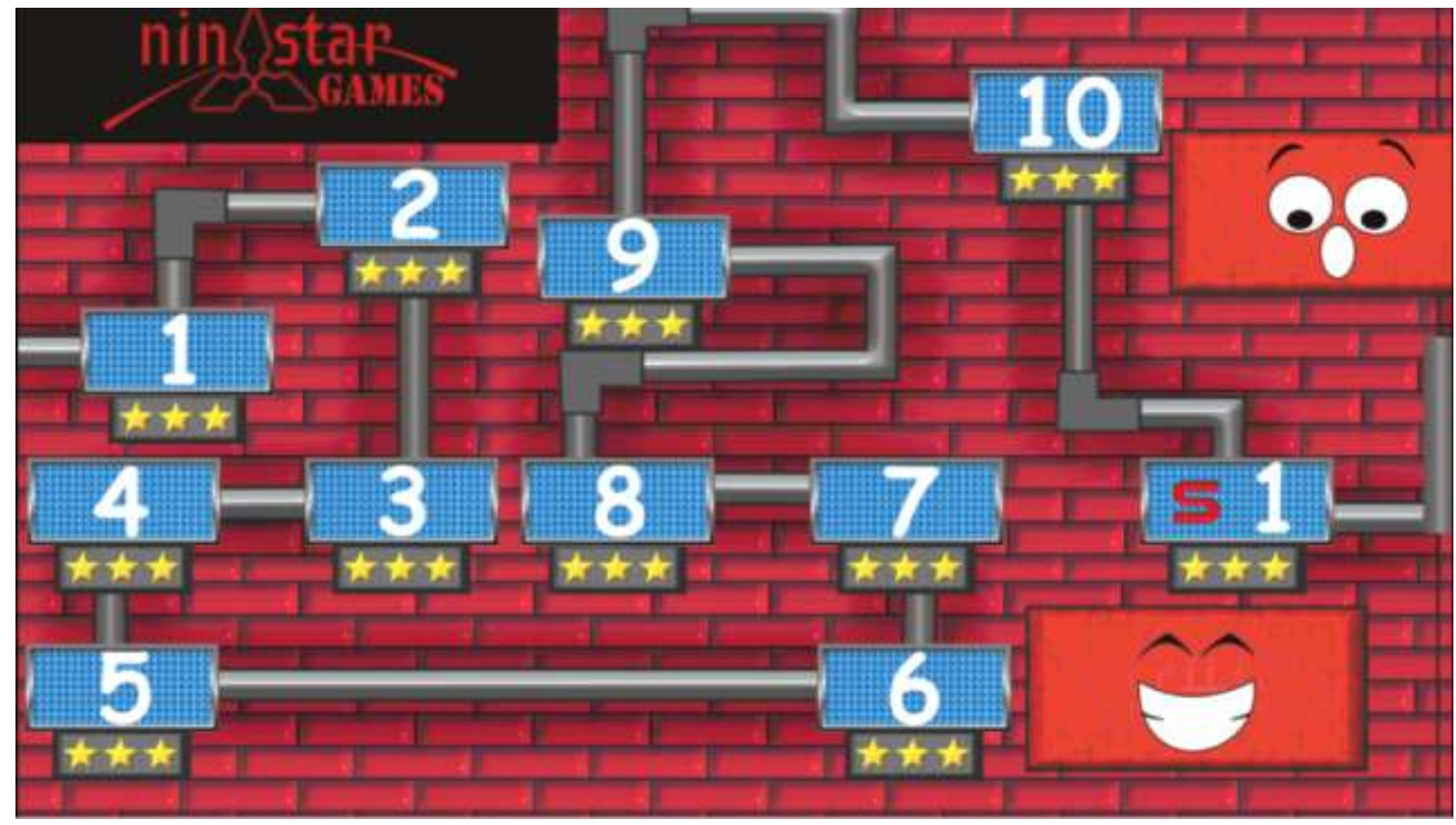

Home screen consist of different levels this game has a total of 33 levels user can toggle between the levels using his finger the camera will follow the finger and display different levels. User has to click on the level to play it and once cleared he can play that level again.

\subsection{Level}

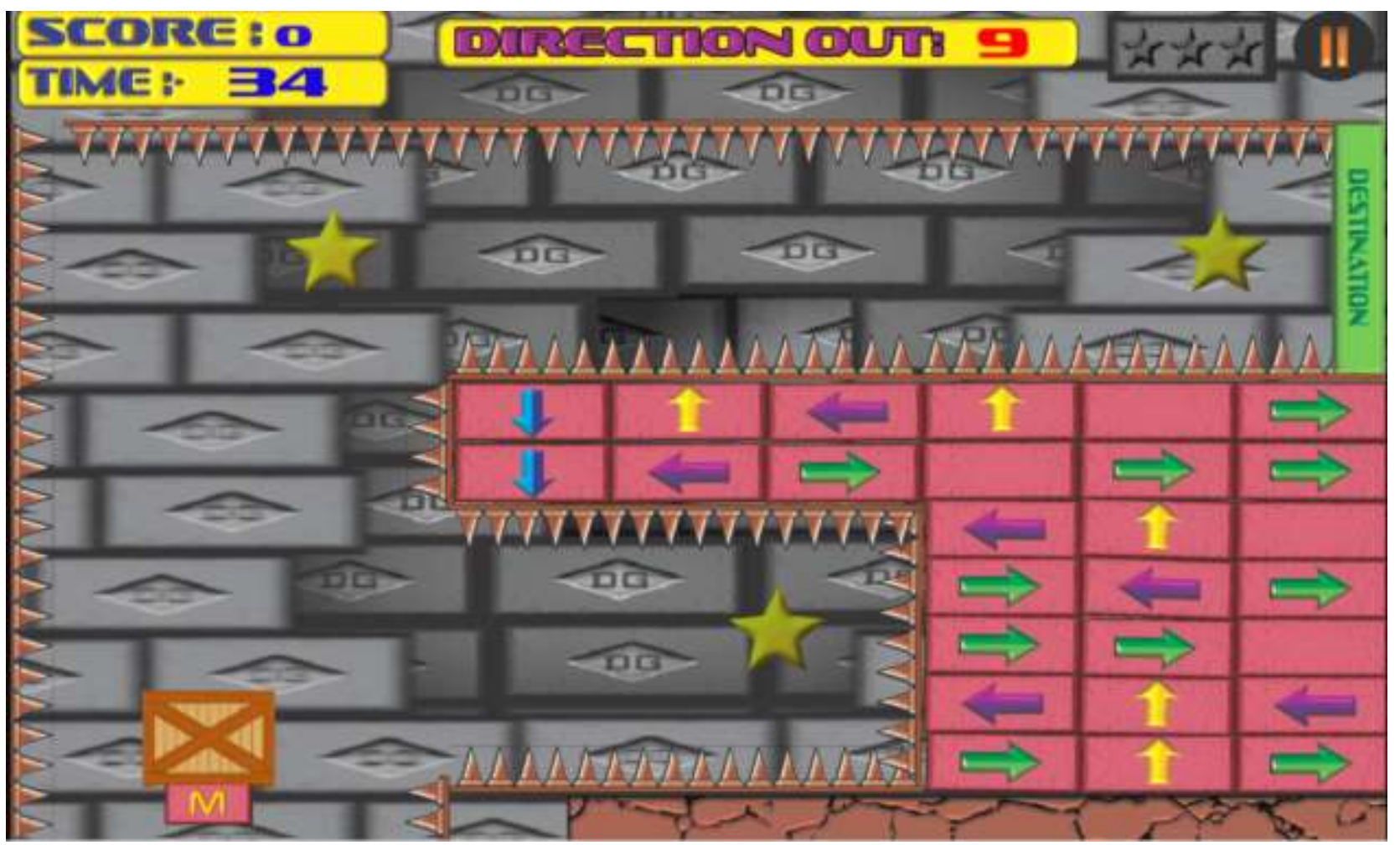


The image above displays one of the level of the game where use has to collect all the stars and reach the destination, the directions on the bricks help the user to manipulate the box, some direction disappears so the user has to remember the position of that particular direction the timer above indicates the disappearance of the directions, the score keeps on increasing on breaking the bricks and collecting the star.

\subsection{Special Level}

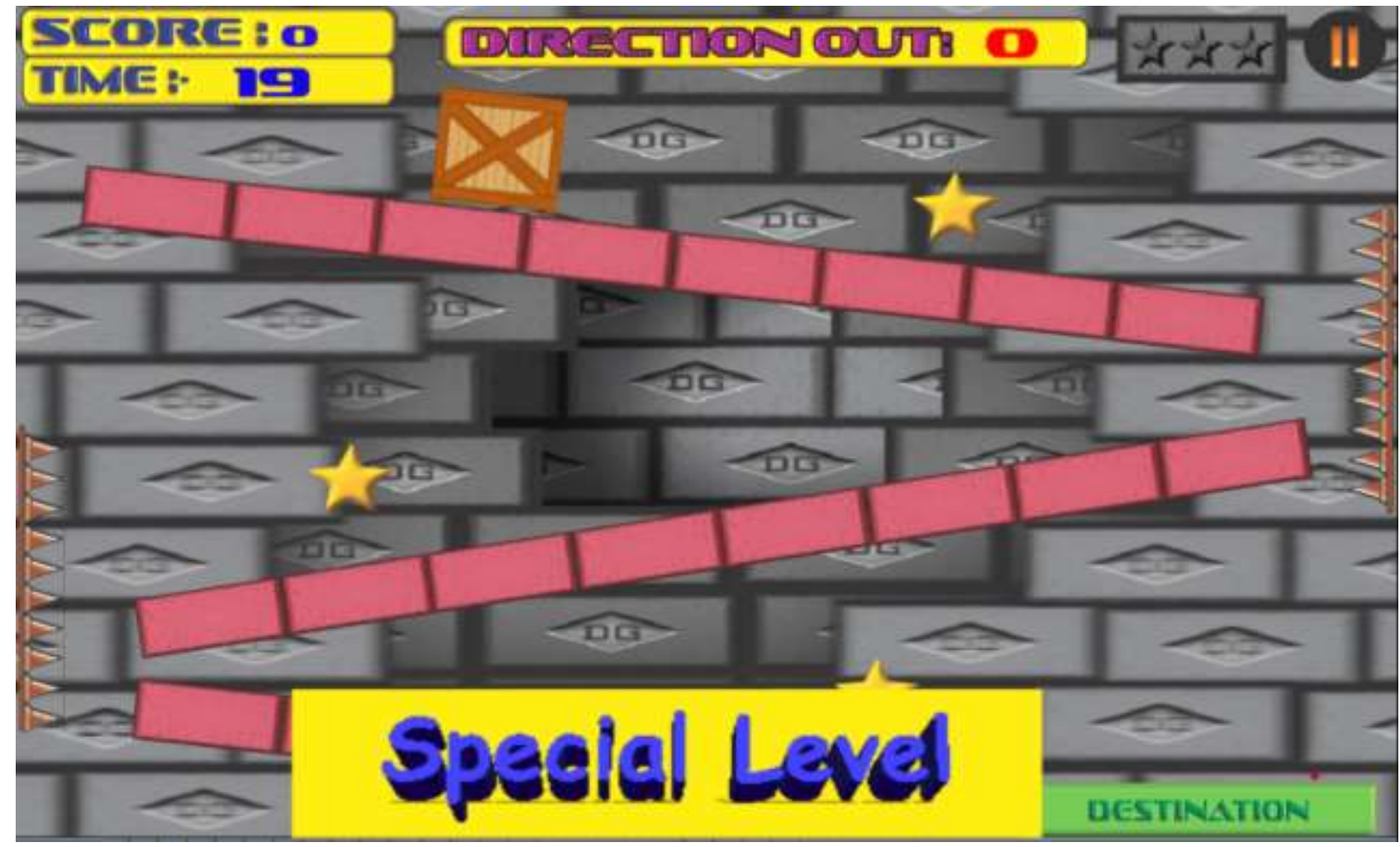

There is also a special level after 10 levels which is completely different from the other levels, each special level has its own challenges and twists.

\section{CONCLUSION}

This paper presents the successful implementation of the android game Breaking Brickz. The levels stated are tested and implemented. Several other levels can be added because of the flexible structure.It reflects on selected challenges and proposes some future directions.

\section{REFERENCES}

[1]. Unity3d.com

[2]. Android Game Programming For Dummies by Derek James

[3]. Beginning Android Games by Robert Green 